\title{
INTELIGENCIA EMOCIONAL Y ANSIEDAD EN MUJERES
}

\author{
Paloma Gil-Olarte \\ Laboratorio de Inteligencia Emocional \\ Universidad de Cádiz (Cádiz, España) \\ paloma.gilolarte@uca.es \\ Rocío Gómez-Molinero \\ Laboratorio de Inteligencia Emocional \\ Universidad de Cádiz (Cádiz, España) \\ Paula Ruiz-González \\ Laboratorio de Inteligencia Emocional \\ Universidad de Cádiz (Cádiz, España) \\ Instituto de Investigación e Innovación \\ Biomédica de Cádiz (INIBICA) (Cádiz, España) \\ Rocío Guil \\ Laboratorio de Inteligencia Emocional \\ Universidad de Cádiz (Cádiz, España)
}

Fecha de Recepción: 20 Enero 2019

Fecha de Admisión: 30 Abril 2019

\section{RESUMEN}

El Informe de la Cuarta Conferencia Mundial sobre la Mujer (Naciones Unidas, 1995) declara el derecho a disfrutar de plena salud física y mental, entendida como un estado de bienestar emocional, social y físico. Siguiendo estos principios, la Fundación Municipal de la Mujer del Ayuntamiento de Cádiz promueve diversas actividades para mejorar la calidad de vida de las mujeres gaditanas, favoreciendo su desarrollo personal y social. En este sentido, el Laboratorio de Inteligencia Emocional de la Universidad de Cádiz colabora con la Fundación de la Mujer realizando un proyecto educativo de Socialización Emocional que potencie un mayor bienestar de las mujeres gaditanas. El objetivo de este trabajo es confirmar si los niveles de ansiedad, considerada como estado y como rasgo, de una muestra de mujeres gaditanas $(N=60)$ mejora tras la realización de un programa basado en el Modelo de Inteligencia Emocional (Mayer y Salovey, 1997). Los resultados presentan una mejora estadísticamente significativa en las dimensiones del Cuestionario Ansiedad EstadoRasgo (STAI) (Spielberger, Gorsuch y Lushene, 1988; versión española de Buela-Casal, GuillénRiquelme y Seisdedos, 2011), aun controlando variables como la edad, estado civil o situación laboral. La importancia de desarrollar las competencias emocionales propias del Modelo de Mayer y Salovey (1997) para disminuir la ansiedad ha sido evidenciada en nuestra investigación 


\section{INTELIGENCIA EMOCIONAL Y ANSIEDAD EN MUJERES}

Palabras claves: inteligencia emocional; competencias emocionales; programas educativos; ansiedad; calidad de vida; bienestar

\section{ABSTRACT}

Emotional intelligence and anxiety in women. The Report of the Fourth World Conference on Women (United Nations, 1995) declares the right to enjoy full physical and mental health, understood as a state of emotional, social and physical well-being. Following these principles, the Women s Foundation of Cádiz Government promotes different activities to improve the quality of life of the women from this city, favouring their personal and social development. In this sense, the Emotional Intelligence Laboratory of the University of Cádiz collaborates with the Foundation of Women, developing an educational program of Emotional Socialization that promotes a greater wellbeing of these women. In this study, our objective is to confirm if the levels of anxiety, considered as a state and as a trait, of a sample of women from Cádiz $(\mathrm{N}=60)$ improve after a training program based on the Emotional Intelligence Model (Mayer and Salovey, 1997). The results show a statistically significant improvement in the dimensions of the State-Trait Anxiety Questionnaire (STAI) (Spielberger, Gorsuch and Lushene, 1988, Spanish version of Buela-Casal, Guillén-Riquelme y Seisdedos, 2011), with no influence of variables such as age, marital status or employment status. The importance of developing emotional competencies from Mayer and Salovey Model (1997) to reduce anxiety has been evidenced in our research

Keywords: emotional intelligence; emotional competencies; educational programs; anxiety; quality of life; well-being

\section{ANTECEDENTES}

El Informe de la Cuarta Conferencia Mundial sobre la Mujer (Naciones Unidas, 1996) declara el derecho a disfrutar de plena salud física y mental, entendida como un estado de bienestar mental, emocional, social y físico. Sin embargo, la prevalencia de los trastornos mentales continúa aumentando, causando efectos considerables en la salud de las personas, su bienestar y calidad de vida.

A nivel mundial y nacional, encontramos que los trastornos ansiosos se encuentran entre las enfermedades mentales más frecuentes en la población general. La Organización Mundial de la Salud (OMS, 2017) indica que en la última década se ha incrementado en un $15 \%$ el número de personas que a nivel mundial padecen trastornos de ansiedad, más de 260 millones de personas afectadas, siendo las mujeres las que presentan el doble de prevalencia que los hombres. En España, la Encuesta Nacional de Salud de 2017 (Ministerio de Sanidad, Consumo y Bienestar Social, 2018) señala que el 6,7\% de los adultos refiere padecer ansiedad crónica, un 4,3\% de los hombres y un $9,1 \%$ de las mujeres.

En esta línea, podemos indicar que las mujeres suelen experimentar mayores niveles y desórdenes de ansiedad en general (Bender, Reinholdt-Dunne, Esbjørn y Pons, 2012; Caballo et al., 2014; McLean, Asnaani, Litz y Hofmann, 2011), siendo la regulación emocional una de las causas que podrían explicar estas diferencias en los niveles de ansiedad entre mujeres y hombres (Aldao, NolenHoeksema y Schweizer, 2010; NolenHoeksema, 2012).

El término ansiedad hace referencia a una reacción emocional que surge cuando las personas se enfrentan a situaciones desconocidas (Lazarus y Folkman, 1984). No obstante, Spielberger $(1966,1972,1989)$ considera que para conceptualizar la ansiedad correctamente hay que diferenciar entre la ansiedad como un rasgo de personalidad y como un estado emocional. Según Spielberger (1966, 1972), la ansiedad-estado, es un estado emocional inmediato y transitorio que se puede modificar en el tiempo y es percibido como una combinación de tensión, aprensión y nerviosismo, preocupaciones, junto a cambios fisiológicos. Por su parte, la ansiedad-rasgo hace refe- 
rencia a un comportamiento predispuesto del individuo a percibir un amplio número de situaciones como amenazantes, que es permanente en el tiempo, siendo éste una disposición, tendencia 0 rasgo. A diferencia de la ansiedad estado, la ansiedad-rasgo no se manifiesta directamente y debe ser inferida por la frecuencia con la que un individuo experimenta aumentos en su estado de ansiedad. En este sentido, las personas con alto grado de ansiedad-rasgo están más predispuestos a sufrir ansiedad-estado de forma más frecuente 0 con mayor intensidad.

Por otro lado, la Inteligencia Emocional se ha definido como "la capacidad de percibir, valorar y expresar emociones adecuada y adaptativamente; la capacidad de acceder y/o generar sentimientos que faciliten el pensamiento, las actividades cognitivas y la acción adaptativa; la capacidad de comprender emociones y el conocimiento emocional; y la capacidad de regular las emociones en uno mismo y en otros para promover el crecimiento emocional e intelectual" (Mayer y Salovey, 1997, p. 10).

En las últimas décadas, el estudio de la Inteligencia Emocional ha generado un gran interés entre la comunidad científica por sus potenciales implicaciones beneficiosas sobre múltiples variables de adaptación personal y social (Martínez, Piqueras, Ramos, 2010), destacando como un importante factor protector contra la ansiedad.

En sentido general, dentro del ámbito de la salud, puntuaciones altas en los niveles de Inteligencia Emocional se relacionan con una mayor salud mental, una adaptación emocional más ajustada y una mejor calidad de vida y bienestar personal (Azpiazu, Esnaola y Sarasa, 2015; Cejudo, 2016; Limonero, Fernández-Castro, Soler-Oritja y Álvarez-Moleiro, 2015; Mikolajczak et al., 2015).

Asimismo, también existen claras relaciones entre la Inteligencia Emocional y la ansiedad. Rodríguez Biglieri, Aune y Vetere (2012) concluyen que existe una relación entre la práctica de estrategias de regulación emocional desadaptativas y la aparición de trastornos de ansiedad. Sin embargo, el manejo de competencias emocionalmente ajustadas favorece la reducción progresiva de la ansiedad (Gutiérrez y Expósito, 2015). Lizeretti y Rodríguez (2011) señalan que aquellas personas que muestran niveles altos de atención emocional y bajos de claridad y reparación emocional se relacionan con síntomas de ansiedad; mientras que aquellas personas que obtienen bajas puntuaciones en reparación emocional y altos en atención emocional se relacionan con el trastorno de ansiedad generalizada.

Sin embargo, actualmente, todavía destaca la necesidad de conocer cuánto y de qué manera la Inteligencia Emocional contribuye a la adaptación en contextos sociales reales (Matthews, Zeidner, y Roberts, 2007). Por este motivo, consideramos necesario desarrollar las competencias emocionales en el contexto de la vida cotidiana en aras de mejorar los niveles de ansiedad que padecen las mujeres gaditanas y favorecer su desarrollo personal y calidad de vida.

\section{OBJETIVOS}

- El primer objetivo, es la elaboración de un programa basado en el Modelo de Capacidades de Mayer y Salovey (1997).

- El segundo objetivo y, principal de este estudio, es conocer si el entrenamiento en las competencias emocionales a través de un programa basado en el Modelo de Capacidades de Mayer y Salovey (1997) disminuye los niveles de ansiedad de una muestra de mujeres

- El tercer objetivo es examinar si las variables de control, rango de edad, estado civil, situación laboral influyen en los niveles de ansiedad de una muestra de mujeres.

\section{PARTICIPANTES}

La muestra está formada por 60 mujeres naturales de la ciudad de Cádiz que acuden a los programas de formación de la Fundación Municipal de la Mujer del EXcmo. Ayuntamiento de Cádiz. 
La edad media del grupo es de 46,07 años con un rango 26-71 y una desviación típica de 11,065. En cuanto al estado civil de la muestra, 8 participantes están divorciadas 0 separadas, 11 viudas, 12 solteras y 29 casadas. Respecto al nivel educativo, un $36,6 \%$ de la muestra ha completado estudios superiores, un $44,1 \%$ de las participantes se ha formado en estudios de ciclo medio y un $13,3 \%$ ha completado los estudios básicos. A pesar del alto nivel de cualificación, el 50\% de las mujeres se encuentra en situación de desempleo, el 33,33\% desempeña un trabajo cualificado y el $16,6 \%$ restante son jubiladas o pensionistas.

Tabla 1. Características sociodemográficas de la muestra

\begin{tabular}{lcc}
\hline VARIABLES & \multicolumn{2}{c}{ PARTICIPANTES } \\
SOCIODEMOGRÁFI & $\mathrm{N}$ & $\%$ \\
\cline { 2 - 3 } CAS & 7 & 11,66 \\
\hline * Edad & 11 & 18,33 \\
- Menos de 30 años & 23 & 38,33 \\
- Entre 31 y 40 años & 11 & 18,33 \\
- Entre 41 y 50 años & 8 & 13,33 \\
- Entre 51 y 60 años & 12 & \\
- Más de 60 años & 29 & 20 \\
\hline * Estado civil & 8 & 48,33 \\
- Soltera & 11 & 13,33 \\
- Casada & & 18,33 \\
- Divorciada o separada & 8 & 13,33 \\
- Viuda & 20 & 33,33 \\
\hline * Nivel educativo & 22 & 36,66 \\
- Estudios básicos & & \\
- Estudios medios & 20 & 33,33 \\
- Estudios superiores & 30 & 50 \\
\hline * Situación laboral & 10 & 16,66 \\
- Trabajo remunerado & & \\
- Desempleo & Jubilación &
\end{tabular}

\section{MÉTODO}

\section{Diseño de la investigación}

La metodología de nuestra investigación sigue un diseño cuasi-experimental, concretamente, un diseño pretest - postest.

\section{Instrumento}

Cuestionario de Ansiedad Estado-Rasgo (State-Trait Anxiety Inventory, STAI, Spielberger, Gorsuch, y Lushene, 1988).

El "Cuestionario de Ansiedad Estado-Rasgo" ha sido adaptado al español por la Editorial TEA. El STAI comprende escalas separadas de autoevaluación que miden dos conceptos independientes: estado y rasgo.

Ansiedad - estado (A-E): es el nivel de ansiedad en un momento determinado, en función de una situación concreta, no de rasgos de personalidad del sujeto, y se considera como un estado o con- 
dición emocional transitoria. Se caracteriza por sentimientos subjetivos de tensión y aprensión, así como la hiperactividad del Sistema Nervioso Autónomo y puede variar con el tiempo y fluctuar en intensidad.

Ansiedad - rasgo (A-R): se define como una propensión ansiosa relativamente estable que diferencia los individuos en su tendencia a percibir las situaciones como amenazadoras y a elevar, consecuentemente, su ansiedad - estado. La ansiedad - rasgo es similar a los constructos llamados "motivos" o "disposiciones que permanecen latentes hasta que son activadas por algunos estímulos de la situación", o bien "disposiciones comportamentales adquiridas".

La consistencia interna de esta escala es $\alpha=0.93$ en la escala Ansiedad-Estado y $\alpha=0,87$ en la escala Ansiedad-Rasgo. En nuestra muestra la fiabilidad total del cuestionario es de $\alpha=0.94$, siendo concretamente en la escala Ansiedad-estado de $\alpha=0.91$ y en la de ansiedad-rasgo de $\alpha=0.90$.

\section{ELABORACIÓN DEL PROGRAMA DE ENTRENAMIENTO EN LAS COMPETENCIAS EMOCIONALES}

La finalidad de este programa es el desarrollo de las competencias socioemocionales con el fin de potenciar la adaptación social de las mujeres gaditanas favoreciendo su conocimiento y crecimiento emocional. Las competencias emocionales son un conjunto de capacidades que permiten comprender, expresar y regular de forma apropiada los fenómenos emocionales, lo que facilita el desenvolverse mejor en las distintas circunstancias de la vida diaria. Las emociones pueden surgir en momentos inoportunos o con una intensidad incorrecta, de manera que perturbe la adaptación al ambiente (Ivcevic y Brackett, 2014; Jacobs y Gross, 2014). Por lo tanto, desde un punto de vista adaptativo, es necesario un proceso que permita una adecuada regulación de las emociones. Por eso, se trabaja en la adquisición y asimilación de conocimientos teóricos y prácticos sobre las emociones básicas que experimentan las mujeres, las situaciones que las provocan, sus formas básicas de manifestación, las conductas que generan. Y lo más importante, se desarrollan diversas estrategias que permitan resolver las situaciones de manera más adaptativa.

En el diseño del programa para el entrenamiento en las competencias emocionales, nos basamos en el Modelo de Inteligencia Emocional de Mayer y Salovey (1997). Mayer, Salovey y Caruso $(2004,2008)$ argumentan que la Inteligencia Emocional se define mejor como una habilidad de la misma manera que la inteligencia cognitiva se conceptualiza como una habilidad. En este sentido, si consideramos la inteligencia como un conjunto de capacidades que permiten resolver problemas, la Inteligencia Emocional podría explicarse como un conjunto de capacidades que permite procesar cognitivamente y utilizar la información emocional para gestionar problemas adaptativamente (Guil, Gil-Olarte, Mestre, Núñez y Guil, 2006).

En esta conceptualización, las habilidades y destrezas de la Inteligencia Emocional se dividen en cuatro ramas: la capacidad de percibir emociones, la capacidad de usar las emociones para facilitar el pensamiento, la capacidad de comprender las emociones y la capacidad de manejar las emociones. Cada una de las habilidades que forman cada rama posee un desarrollo progresivo y jerárquico que comienza con las destrezas más básicas hasta las más complejas.

Brevemente, podemos decir que la primera rama, Percepción valoración y expresión de las propias emociones, implica la capacidad para identificar y reconocer tanto los sentimientos propios como los de los demás. La segunda rama, Facilitación emocional de las actividades cognitivas; uso inteligente de las emociones, hace referencia a la capacidad para tener en cuenta los sentimientos cuando razonamos o tomamos decisiones. La tercera rama, Comprensión y análisis de la información emocional y empleo del conocimiento emocional, supone la capacidad para comprender la información emocional, cómo se combinan y evolucionan los estados emocionales. Por último, la cuarta rama, Regulación emocional; conducción de las emociones, es la capacidad para estar abier- 
tos a los estados emocionales positivos y negativos, aprovechar la información que nos ofrecen para gestionar las emociones en uno mismo y los demás.

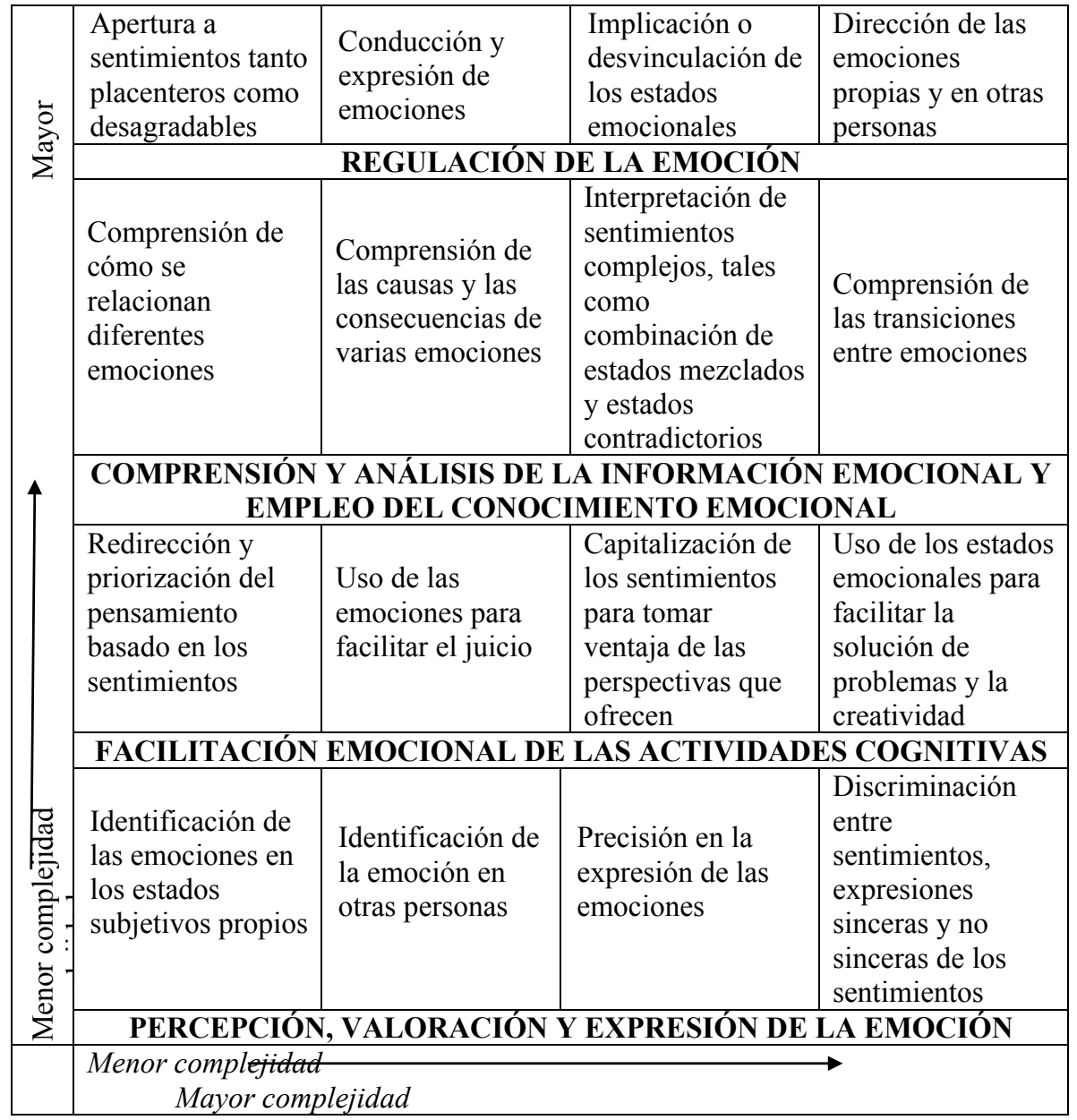

Teniendo presente estos objetivos, nos decantamos por trabajar a través de las técnicas de grupo. Consideramos que éstas permiten generar un clima muy favorable, al tiempo que propicia que afloraren multitud de emociones en las participantes, así como situaciones emocionales que, desde nuestro punto de vista, resultan imprescindibles cuando pretendemos adiestrarnos en la adquisición y automatización de habilidades cognitivas de procesamiento de información emocional.

Se realiza una programación de 16 sesiones con una duración de 2 horas cada una que permite aprender las diversas habilidades incluidas en cada rama y que se plantean de manera progresiva desde las habilidades más sencillas a las más complejas, mientras se refuerzan las ya adquiridas. 
De esta manera se pretende consolidar el entrenamiento en habilidades de manejo de información emocional.

\section{RESULTADOS}

Una vez elaborado nuestro programa de entrenamiento, comprobamos nuestro segundo objetivo, "conocer si el entrenamiento en las competencias emocionales a través de un programa basado en el Modelo de Capacidades de Mayer y Salovey (1997).

Tanto la Ansiedad-Rasgo como la Ansiedad-Estado se vieron reducidas de manera estadísticamente significativa tras el entrenamiento en habilidades cognitivas de procesamiento de la información. Así, al finalizar el programa, se registró una reducción en la tendencia de las participantes a percibir las situaciones como amenazadoras, así como en su propensión a estados emocionales ansiosos relativamente estables (Ansiedad-Rasgo). Igualmente, se observó una disminución en la experimentación de sentimientos subjetivos de tensión y aprensión, y en la hiperactividad del sistema nervioso autónomo de nuestra muestra (Ansiedad-Estado).

Gráfica 1. STAI-AE/STAI-AR pretest-postest

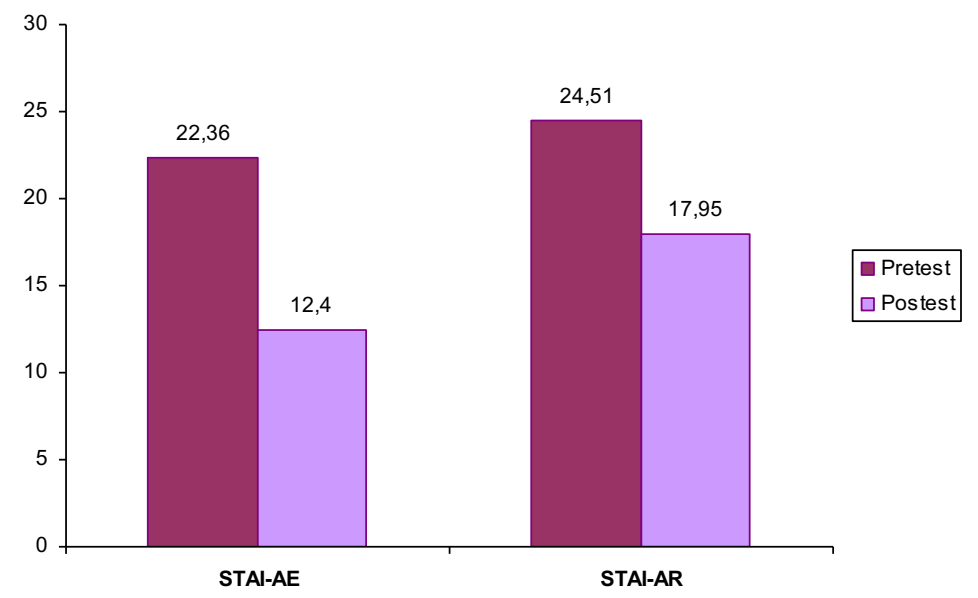

Como podemos observar al comparar las gráficas pretest - postest, estos resultados confirman la hipótesis que nos planteábamos al comenzar la aplicación del programa, ya que los valores de la ansiedad han mejorado en todos sus niveles. En concreto, observamos como la ansiedad disminuye alrededor de un $50 \%$.

A la hora de dar respuesta a nuestro tercer objetivo "Examinar si las variables de control, rango de edad, estado civil, situación laboral influyen en los niveles de ansiedad de una muestra de mujeres" realizamos la prueba one-way MANOVA en las variables de control evaluadas.

Observamos que no existen diferencias significativas entre las mujeres antes del entrenamiento de las competencias socioemocionales con respecto al estado civil, $F(89,171)=1,210, p>, 005$ ( $p$ $=, 145)$; Wilk's $=0,230$, parcial $2=, 39$. Tampoco encontramos diferencias con respecto al rango de edad $F(119,225)=1,002, p>, 005(p=, 488)$; Wilk's $\lambda=0,183$, parcial $\varepsilon 2=, 34$. De la misma manera, tampoco encontramos diferencias significativas en relación a la situación laboral de la muestra $F(59,116)=1.629, p>, 005(p=, 013$; Wilk's $\lambda=0,295$, parcial $\varepsilon 2=, 46$. 


\section{INTELIGENCIA EMOCIONAL Y ANSIEDAD EN MUJERES}

Finalmente, para comprobar la posible influencia que han ejercido las variables de control sobre las variables de adaptación al finalizar el programa, volvemos a realizar la prueba one-way MANOVA en las variables evaluadas en el postest de nuestro grupo experimental.

En este caso, tampoco existen diferencias significativas entre las mujeres que ha recibido en programa teniendo en cuenta su estado civil $F(86,84,66)=1,275, p>, 005(p=, 132)$; Wilk's $\lambda=$ 0,82 , parcial $\varepsilon 2=, 56$; su situación laboral $F(57,58)=, 781, p>, 005(p=, 826)$; Wilk's $\lambda=0,315$, parcial $\varepsilon 2=, 44$; 0 su rango de edad $F(115,109,887)=1,842, p>, 005(p=, 488)$; Wilk's $\lambda=0,014$, parcial $\varepsilon 2=, 66$.

De una manera gráfica, podemos observar cómo las mejoras en las variables de adaptación son significativas y no existen diferencias en la ansiedad estado y rasgo cuando tenemos en cuenta el estado civil, el rango de edad o la situación laboral de las mujeres.

En la escala de Ansiedad-Estado, que muestra el nivel de ansiedad en un momento concreto, encontramos que las mujeres que informan un mayor nivel son en primer lugar las separadas, en segundo lugar, las solteras con una puntuación muy parecida a las viudas y las que poseen un menor grado de ansiedad-estado son las casadas. Resultados que se repiten en la medida postest aunque con niveles inferiores de ansiedad.

Si consideramos el rango de edad, las puntuaciones superiores corresponden a las mujeres entre 31 y 40 años, seguidas de las de 51 a 60 años. Por otro lado, las participantes que se encontraban en situación de desempleo, muy seguido de las mujeres con trabajo remunerado, tienen niveles superiores en la ansiedad-estado. Al finalizar el entrenamiento, las mujeres han disminuido significativamente su ansiedad, destacando principalmente las mujeres con edades comprendidas entre los 31 y 40 y las mujeres desempleadas.

Gráfica 2. STAI-AE pretest-postest

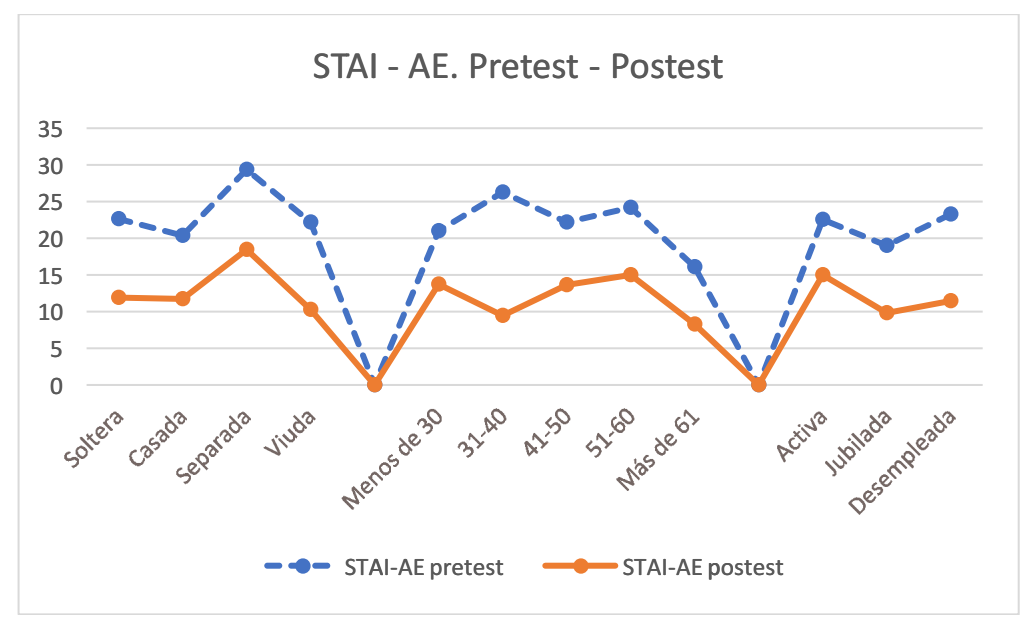

Cuando evaluamos la Ansiedad-Rasgo, definida como una propensión ansiosa relativamente estable, verificamos una clara disminución de la misma y observamos cómo las puntuaciones más altas, tanto en el pretest como en el postest, se hallan en las mujeres solteras seguidas de las separadas, viudas y casadas; en las menores de 30 años y las que tienen entre 51 y 60; y en las mujeres con un trabajo remunerado seguidas de las desempleadas. 
Gráfica 3. STAI-AR pretest-postest

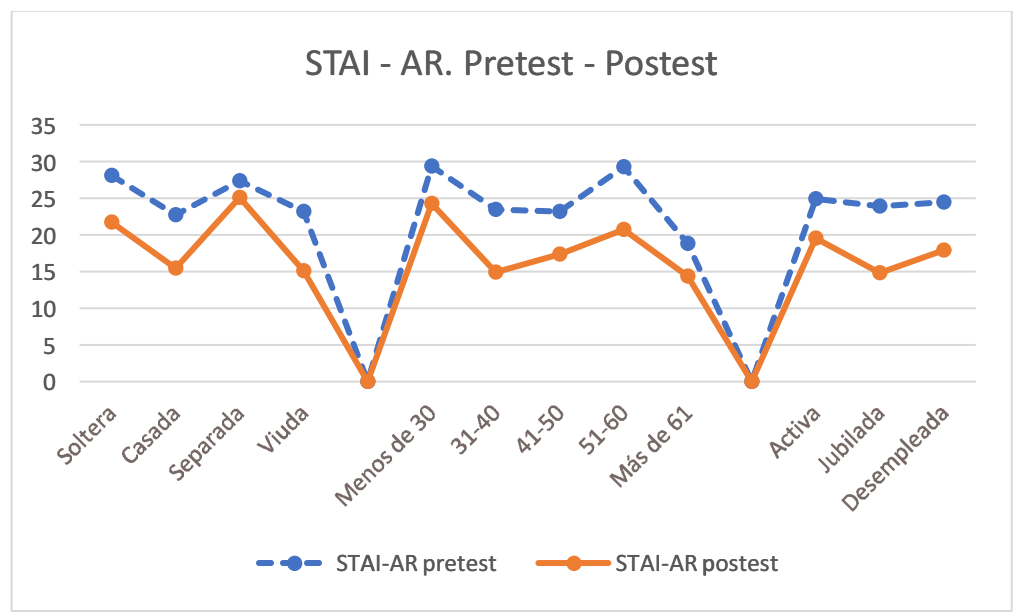

\section{CONCLUSIONES}

En nuestra muestra observamos una mejoría en los niveles de ansiedad. Los resultados de nuestra investigación ofrecen una posible explicación a esta situación. Entendemos que las mujeres han mejorado su capacidad de identificar y comprender sus propios estados emocionales, y esto les ha permitido gestionar de manera más adaptativa las emociones negativas y mantener las emociones positivas. Concretamente, al favorecer estas capacidades emocionales, las mujeres han aprendido a saber cómo, por qué y en qué sentido afectan las emociones a sus conductas y pensamientos, de manera que poseen los instrumentos necesarios para regular sus emociones y comportamientos y aumentar su capacidad para adecuarse a la situación. Así, tras el entrenamiento, las mujeres consiguieron disminuir la percepción de amenaza de ciertas situaciones estresantes, la experimentación de sentimientos subjetivos de tensión y aprensión y, consecuentemente, lograron reducir la propensión a estados emocionales ansiosos relativamente estables.

Finalmente, en relación al análisis de las variables control, aunque tampoco forman parte de nuestro objetivo fundamental, nos gustaría concluir que el estado civil, el rango de edad y la situación laboral de la muestra no afecta a la variable de adaptación contemplada en el estudio.

Estas evidencias sugieren que debido al entrenamiento de las competencias emocionales incluidas en el Modelo de Mayer y Salovey (1997), las mujeres han desarrollado su capacidad para atender a sus estados afectivos, reconocer correctamente sus sentimientos y reparar sus emociones, lo que favorecerá la disminución de sus niveles de ansiedad estado y rasgo y, por tanto, la mejora de su bienestar personal y calidad de vida. En definitiva, consideramos justificada no sólo la necesidad, sino también la importancia del diseño y puesta en marcha de programas de educación socioemocional (Gil-Olarte et al., 2006; Guil y Gil-Olarte, 2007) para mejorar los niveles de ansiedad y, por tanto, la calidad de vida.

\section{REFERENCIAS BIBLIOGRÁFICAS}

Aldao, A., Nolen-Hoeksema, S., \& Schweizer, S. (2010). Emotion-regulation strategies across psychopathology: A meta-analytic review. Clinical Psychology Review, 30(2), 217-237.

Azpiazu, L., Esnaola, I. y Sarasa, M. (2015). Predictive capacity of social support on emotional inte- 
Iligence in adolescence. European Journal of Education and Psychology, 8(1), 23-29.

Bender, P. K., Reinholdt-Dunne, M. L., Esbjørn, B. H., \& Pons, F. (2012). Emotion dysregulation and anxiety in children and adolescents: Gender differences. Personality and Individual Differences, 53(3), 284-288.

Buela-Casal, G. Guillén-Riquelme, A. y Seisdedos, N. (2011). STAI: Cuestionario de Ansiedad Estado-Rasgo. Adaptación española (8ª ed.). Madrid: TEA Ediciones.

Caballo, V. E., Salazar, I. C., Irurtia, M. J., Arias, B., Hofmann, S. G., \& CISO-A Research Team. (2014). Differences in social anxiety between men and women across 18 countries. Personality and Individual Differences, 64, 35-40.

Cejudo, J. (2016). Relationship between Emotional Intelligence and mental health in School Counselors. Electronic Journal of Research in Educational Psychology, 14(1), 131-154.

Gil-Olarte, P., Guil, R., Mestre, J.M., Núñez, I. y Guil, A. (2006). Women s emotional socialization program. En A. L. Comunian y R. Rith (Eds), International Perspectives in Psychology, (pp. 166176). London: Psychology Press LTD.

Guil, R. y Gil-Olarte, P. (2007). Inteligencia Emocional y Educación: desarrollo de competencias socioemocionales. En J. M. Mestre y P. Fernández-Berrocal (Coords.), Manual de Inteligencia Emocional (pp. 189-215). Madrid: Ediciones Pirámide.

Gutiérrez, M. y Expósito, J. (2015). Autoconcepto, dificultades interpersonales, habilidades sociales y conductas asertivas en adolescentes. Revista Española de Orientación y Psicopedagogía, 26(2), 42-58.

Ivcevic, Z., \& Brackett, M. (2014). Predicting school success: Comparing Conscientiousness, Grit, and Emotion Regulation Ability. Journal of Research in Personality. 52, 29-36.

Jacobs, S. E., \& Gross, J. J. (2014). Emotion regulation in education: Conceptual foundations, current applications, and future directions. En R. Pekrun \& L. Linnenbrink-Garcia (Eds.), Educational psychology handbook series. International handbook of emotions in education (pp. 183-201). New York: Routledge/Taylor \& Francis Group.

Lazarus, R. S., \& Folkman, S. (1984). Coping and adaptation. En W. D. Gentry (Ed.), The Handbook of Behavioral Medicine (pp. 282-325.). New York: Guilford.

Limonero, J.T., Fernández-Castro, J., Soler-Oritja, J. y Álvarez-Moleiro, M. (2015). Emotional intelligence and recovering from induced negative emotional state. Frontiers in Psychology, 6, 1-8.

Lizeretti, N. P. y Rodríguez, A. (2011). La inteligencia emocional en salud mental: Una revisión. Ansiedad y Estrés, 17, 233-253.

Martínez, A. E., Piqueras, J. A. y Ramos, V. (2010). Inteligencia emocional en la salud física y mental. Electronic Journal of Research in Educational Psychology. 21, 8(2), 861-890.

Matthews, G., Zeidner, M., \& Roberts, R. D. (2007). The science of emotional intelligence: Knowns and unknowns. Oxford: Oxford University Press.

Mayer, J. D. y Salovey, P. (1997). What is emotional intelligence? En P. Salovey y D. J. Sluyter (Eds.), Emotional development and emotional intelligence educational implications (pp. 3-34). New York: Basic Books.

Mayer, J. D., Salovey, P., \& Caruso, D. (2004). Emotional intelligence: Theory, Findings and Implications. Psichological Inquiry, 15, 197-215.

Mayer, J. D., Salovey, P., \& Caruso, D. (2008) Emotional intelligence. New ability or eclectic tratis? American Psychologist, 63(6), 503-517.

McLean, C. P., Asnaani, A., Litz, B. T., \& Hofmann, S. G. (2011). Gender differences in anxiety disorders: Prevalence, course of illness, comorbidity and burden of illness. Journal of Psychiatric Research, 45(8), 1027-1035. 
Mikolajczak, M., Avalosse, H., Vancorenland, E., Verniest, R., Callens, M., van Broeck, N., \& Mierop, A. (2015). A nationally representative study of emotional competence and health. Emotion, 15(5), 653-667.

Ministerio de Sanidad, Consumo y Bienestar Social (2018). Encuesta Nacional de Salud 2017. Madrid: Ministerio de Sanidad, Consumo y Bienestar Social.

Naciones Unidas (1996). Informe de la Cuarta Conferencia Mundial sobre la Mujer. Nueva York: Naciones Unidas

Nolen-Hoeksema, S. (2012). Emotion regulation and psychopathology: The role of gender. Annual Review of Clinical Psychology, 8, 161-187.

Organización Mundial de la Salud (2017). Informe del Día Mundial de la Salud.

Rodríguez Biglieri, R., Auné, S., \& Vetere, G. (2012). Regulación emocional en pacientes con trastornos de ansiedad: Un estudio comparativo con una muestra comunitaria. Anuario de Investigaciones, 19(1), 273-280.

Spielberger, C.D. (1966). Theory and research on anxiety. En C.D. Spielberger (Ed.), Anxiety and Behavior (pp. 3-22). New York: Academic Press.

Spielberger, C.D. (1972). Anxiety as an emotional state. En C.D. Spielberger (Ed.), Anxiety Behavior (pp. 23-49). New York: Academic Press.

Spielberger, C.D. (1989). Anxiety in sports: An international perspective. New York: Hemisphere Publishing Corporation.

Spielberger, R. L., Gorsuch, R. L. y Lushene, R. E. (1988). Cuestionario de ansiedad estado-rasgo. Manual. $3^{\mathrm{a}}$ ed. Madrid: TEA. 
